\title{
Dopamine Dynamics during Continuous Intracranial Self- Stimulation: Effect of Waveform on Fast-Scan Cyclic Voltammetry Data
}

\author{
Nathan T. Rodeberg, ${ }^{\dagger}$ Justin A. Johnson, ${ }^{\dagger}$ Elizabeth S. Bucher, ${ }^{\dagger}$ and R. Mark Wightman ${ }^{*}+$ \\ ${ }^{\dagger}$ Department of Chemistry and ${ }^{\star}$ Neuroscience Center and Neurobiology Curriculum, University of North Carolina at Chapel Hill, \\ Chapel Hill, North Carolina 27599-3290, United States
}

\section{Supporting Information}

ABSTRACT: The neurotransmitter dopamine is heavily implicated in intracranial self-stimulation (ICSS). Many drugs of abuse that affect ICSS behavior target the dopaminergic system, and optogenetic activation of dopamine neurons is sufficient to support self-stimulation. However, the patterns of phasic dopamine release during ICSS remain unclear. Early ICSS studies using fast-scan cyclic voltammetry (FSCV) rarely observed phasic dopamine release, which led to the surprising conclusion that it is dissociated from ICSS. However, several advances in the sensitivity (i.e., the use of waveforms with extended anodic limits) and analysis (i.e., principal component regression) of FSCV measurements have made it possible to detect smaller, yet physiologically relevant, dopamine release events. Therefore, this study revisits phasic dopamine release during ICSS using these tools. It was found that the anodic limit of the voltammetric waveform has a substantial effect on the patterns of dopamine release observed during continuous ICSS. While data collected with low anodic limits (i.e., $+1.0 \mathrm{~V})$ support the disappearance of phasic dopamine release observed in previous investigation, the use of high anodic limits $(+1.3 \mathrm{~V},+1.4 \mathrm{~V})$ allows for continual detection of dopamine release throughout ICSS. However, the $+1.4 \mathrm{~V}$ waveform lacks the ability to resolve narrowly spaced events, with the best balance of temporal resolution and sensitivity provided by the $+1.3 \mathrm{~V}$ waveform. Ultimately, it is revealed that the amplitude of phasic dopamine release decays but does not fully disappear during continuous ICSS.

KEYWORDS: Dopamine, voltammetry, intracranial self-stimulation, kinetics
$\mathrm{I}_{\mathrm{ntr}}^{\mathrm{n}}$ ntracranial self-stimulation (ICSS), in which animals are trained to respond for electrical stimulation of the brain, is a central paradigm for investigating brain reward pathways that are activated by drugs of abuse and natural reward-seeking behavior. ${ }^{1-3}$ The neurotransmitter dopamine has long been associated with this task in anatomical ${ }^{4-6}$ and pharmacolog$\mathrm{ical}^{2,7}$ studies. For instance, drugs that target the dopaminergic system, such as amphetamine, cocaine, and other dopamine receptor-specific ligands, alter ICSS behavior even in welltrained animals. ${ }^{2,7,8}$ Furthermore, regions that promote the strongest ICSS response when stimulated contain dopaminergic neurons. ${ }^{6}$ However, the role of direct activation of these neurons has been controversial. ${ }^{9}$ Paired-pulse collision studies of the medial forebrain bundle (MFB) have implicated large, myelinated descending fibers to the ventral tegmental area (VTA) as the principal neuronal population activated with typical electrical stimulation parameters, while suggesting that direct activation of small, unmyelinated dopamine neurons makes only a minor contribution..$^{10,11}$ Instead, separate neurons are thought to activate dopamine cells trans-synaptically during ICSS through the release of excitatory neurotransmitters, such as acetylcholine ${ }^{12}$ and glutamate. ${ }^{13}$ Nevertheless, selective activation of dopaminergic neurons using optogenetics is sufficient to drive self-stimulation behavior. ${ }^{8,14,15}$

Direct measurements of dopamine release during ICSS were first made using microdialysis, which monitors gradual changes in tonic extracellular dopamine levels. This technique typically displays increased dopamine concentrations during ICSS followed by a decline to basal levels following trial termination. ${ }^{16-18}$ The development of fast-scan cyclic voltammetry (FSCV) permitted the measurement of dopamine dynamics on a time scale relevant to behavioral responses (i.e., phasic dopamine release). ${ }^{19-21}$ In contrast to microdialysis measurements, FSCV measurements in the nucleus accumbens (NAc) have revealed a progressive decline in electrically evoked release during continuous ICSS, with no detectable dopamine release present in later periods of ICSS behavior despite long timeouts between behavioral sessions. ${ }^{19}$ This led to the conclusion that phasic dopamine release was not necessary

Received: May 20, 2016

Accepted: August 22, 2016

Published: August 22, 2016 
a

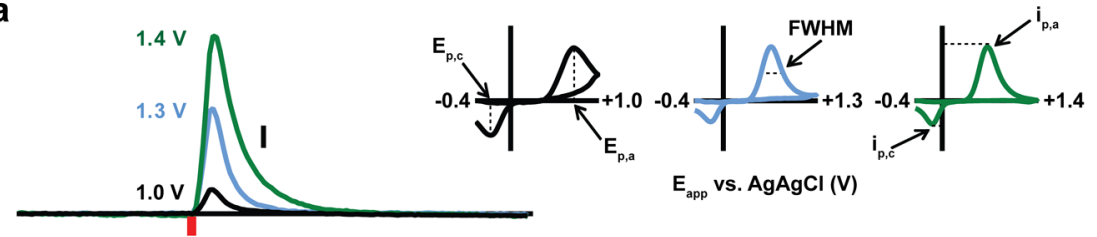

$15 \mathrm{~s}$
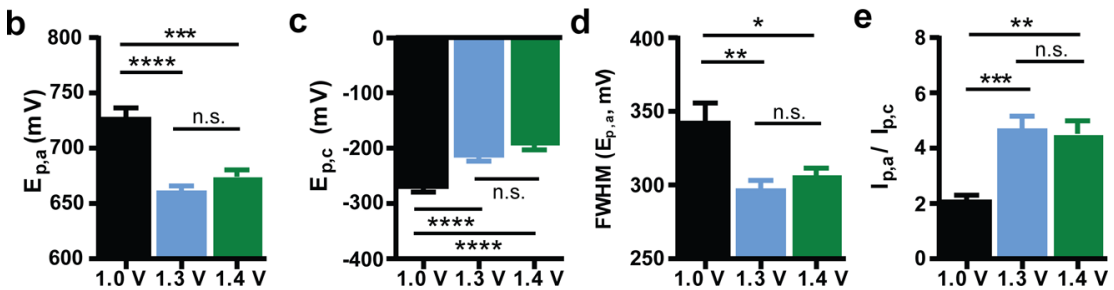

Figure 1. Differences in cyclic voltammogram characteristics across waveforms. (a) Electrically evoked dopamine release measured with each waveform in an anesthetized rat (scale bar $=2 \mathrm{nA}$ ), with inset cyclic voltammograms displayed for each waveform. Voltammogram characteristics of interest are labeled. (b) Anodic peak potentials $\left(E_{\mathrm{p}, \mathrm{a}}\right)$ varied significantly across separate waveform anodic limits at the same electrode $(+1.0 \mathrm{~V}$ waveform, $728 \pm 9 \mathrm{mV}$; +1.3 V waveform, $662 \pm 4 \mathrm{mV}$; $+1.4 \mathrm{~V}$ waveform, $674 \pm 6 \mathrm{mV}$ ), $* * * p<0.001$, $* * * * p<0.0001$. (c) Cathodic peak potentials significantly varied across waveforms $(+1.0 \mathrm{~V}$ waveform, $-273 \pm 6 \mathrm{mV} ;+1.3 \mathrm{~V}$ waveform, $-217 \pm 6 \mathrm{mV} ;+1.4 \mathrm{~V}$ waveform, $-194 \pm 9$ $\mathrm{mV}),{ }^{*} p<0.05$. (d) The full-width at half-maximum (fwhm) for the anodic peak varied significantly across waveforms $(+1.0 \mathrm{~V}$ waveform, $344 \pm 12$ $\mathrm{mV} ;+1.3 \mathrm{~V}$ waveform, $298 \pm 5 \mathrm{mV} ;+1.4 \mathrm{~V}$ waveform, $307 \pm 5 \mathrm{mV}$ ), $* * p<0.01$. (e) The ratio of the peak anodic current to the magnitude of the peak cathodic current varied significantly across waveforms ( $+1.0 \mathrm{~V}$ waveform, $2.15 \pm 0.15 ;+1.3 \mathrm{~V}$ waveform, $4.72 \pm 0.4 ;+1.4 \mathrm{~V}$ waveform, $4.53 \pm$ $0.5)$. Error bars reflect standard error, based on the number of electrodes.

for the maintenance of ICSS behavior, an unexpected finding given previous pharmacological evidence.

However, it remains unclear whether phasic dopamine release was fully abolished or rather fell to undetectable levels. Advances in the field of FSCV have improved the technique's limit of detection (LOD). In the original study, dopamine concentrations were evaluated using univariate analysis, which did not account for contributions from $\mathrm{pH}$ or noise present in the experimental CV. Since then, multivariate calibration has become standard for analyzing voltammetric data collected in awake animals. $^{22-25}$ One such technique, principal component regression (PCR), has been extensively characterized and validated for $\mathrm{FSCV}^{24,25,26,27}$ and is able to separate and quantitate multiple electroactive species of interest while diminishing noise contributions, allowing for more effective isolation of the dopamine signal. ${ }^{26}$

The original measurements made with carbon-fiber microelectrodes used a voltammetric waveform with an anodic limit of $+1.0 \mathrm{~V}$. Subsequent studies have shown that the use of a higher anodic limit promotes the generation of surface oxides that facilitate the adsorption of dopamine, enhancing sensitivity. ${ }^{28,29}$ Additionally, extended anodic limits also provide active and continuous regeneration of the carbon-fiber surface, ${ }^{29}$ permitting the maintenance of high sensitivity throughout the measurement period. When PCR and extended waveforms are combined, the consequent decrease in the LOD may allow the monitoring of smaller dopamine transients previously unobservable with the use of the $+1.0 \mathrm{~V}$ waveform and univariate analysis.

In this study, dopamine fluctuations during continuous ICSS were re-evaluated using three voltammetric waveforms commonly employed in vivo (anodic limits of $+1.0 \mathrm{~V},+1.3 \mathrm{~V}$, and $+1.4 \mathrm{~V}$ vs $\mathrm{Ag} / \mathrm{AgCl}$ ) and PCR. This approach reveals that phasic dopamine release is not abolished during ICSS, but rather decays to smaller, steady-state levels previously undetectable with less sensitive methods. However, higher anodic limits result in diminished temporal resolution that precludes the ability to separate individual transients during rapid self-stimulation and, subsequently, the delineation of how dopamine release changes during this task. Therefore, the waveform utilized considerably impacts the amplitude and time course of voltammetric data collected in freely moving animals. Failure to recognize these differences could lead to a misinterpretation of the role of dopamine in this rewardbased behavior.

\section{RESULTS}

Anodic Limit and Stimulated Release in Anesthetized Animals. Early FSCV measurements of dopamine release in awake animals were made using a voltammetric waveform scanning from a holding potential of $-0.4 \mathrm{~V}$ to an anodic limit of $+1.0 \mathrm{~V} .^{30,31}$ While the resulting sensitivity was adequate to measure real-time dopamine release during early ICSS, ${ }^{19}$ the dopamine signal disappeared after multiple lever presses and did not reappear even following a $30 \mathrm{~min}$ timeout. It was unclear whether this was due to insufficient sensitivity or the absence of dopamine release during these later measurements. Subsequent investigation into the effect of the anodic waveform limit showed increased sensitivity using an anodic limit of +1.4 $\mathrm{V},{ }^{28}$ with an anodic limit of $+1.3 \mathrm{~V}$ later subsequently taken as optimal for dopamine measurements. The relative sensitivities to dopamine of these waveforms are evident in their post vivo calibration factors (normalized to $100 \mu \mathrm{m}$ fiber lengths; $+1.0 \mathrm{~V}$ waveform, $3.9 \pm 0.2 \mathrm{nA} / \mu \mathrm{M}$; $+1.3 \mathrm{~V}$ waveform, $11.8 \pm 0.8 \mathrm{nA} /$ $\mu \mathrm{M} ;+1.4 \mathrm{~V}$ waveform, $28.8 \pm 3.3 \mathrm{nA} / \mu \mathrm{M})$.

While the effect of the anodic limit on dopamine sensitivity has been well characterized in vitro, ${ }^{28,32}$ its effect on temporal responses to dopamine release events in vivo is less clear. To investigate this, measurements of electrically stimulated dopamine release ( 24 pulses, $300 \mu \mathrm{A}, 60 \mathrm{~Hz}, n=6$ for each waveform at each electrode) were made in the NAc shell of anesthetized rats $(n=8)$. Due to the heterogeneity of dopamine release kinetics in the $\mathrm{NAc}^{33}$ measurements were made using all three waveforms at each recording site to enable within-subject comparisons (Figure 1a). Because the extended anodic limits alter the electrode surface, ${ }^{29}$ measurements were 
made in ascending order of anodic limit to prevent effects of prior history on electrode responses. Peak evoked dopamine concentrations $\left([\mathrm{DA}]_{\max }\right)$, rise time (10-90\% max signal), and $t_{1 / 2}$ values $(100-50 \%)$ were compared across waveforms (Table 1). No significant differences in $[\mathrm{DA}]_{\max }$ were seen

Table 1. Comparisons of $[\mathrm{DA}]_{\max }$ Rise Time, and $t_{1 / 2}$ between Different Waveforms ${ }^{a}$

$\begin{array}{cccc}\text { waveform } & {[\mathrm{DA}]_{\max } \mathrm{nM}} & \text { rise time }(10-90 \%), \mathrm{s} & t_{1 / 2}(100-50 \%), \mathrm{s} \\ +1.0 \mathrm{~V} & 267 \pm 64 & 0.3 \pm 0.05 & 0.5 \pm 0.05 \\ +1.3 \mathrm{~V} & 321 \pm 78 & 0.3 \pm 0.02 & 0.7 \pm 0.05 \\ +1.4 \mathrm{~V} & 244 \pm 56 & 0.3 \pm 0.02 & 1.1 \pm 0.08\end{array}$

${ }^{a}$ Data is expressed as mean $\pm \operatorname{SEM}$ ( $n=8$ for each data set).

between waveform (repeated measures one-way ANOVA, $F(2,14)=3.814, p>0.05)$. While differences were not observed in the rise time (10-90\% max signal) for electrically evoked transients across waveforms (repeated measures oneway ANOVA, $F(2,14)=0.1273, p>0.05)$, there were significant differences in $t_{1 / 2}$ values $(100-50 \%)$ (repeated measures one-way ANOVA, $F(2,14)=39.94, p<0.0001)$. Tukey's multiple comparisons post hoc test revealed significant differences between the $+1.4 \mathrm{~V}$ waveform and both the $+1.0 \mathrm{~V}$ and $+1.3 \mathrm{~V}$ waveforms $(p<0.001)$ but not between the $+1.0 \mathrm{~V}$ and +1.3 waveform $(p>0.05)$. The falling portion of the dopamine signal is a measure of the response time of the electrode as well as an index of the uptake rate mediated by the dopamine transporter. The results here demonstrate that the diminished temporal response of higher anodic limit waveforms preferentially affects the uptake-dominated region of electrically stimulated release events, while minimally affecting the region dominated by release.

Differences in the voltammetric characteristics of dopamine across waveforms are also evident. More negative anodic peak locations (Figure 1b), more positive cathodic peak locations (Figure 1c), and smaller anodic peak widths (Figure 1d) were seen with extended waveforms compared with the $+1.0 \mathrm{~V}$ waveform. These observations are consistent with enhanced electron transfer kinetics, ${ }^{34}$ likely due to generation of surface oxide groups. Consistent with a previous study showing differences in voltammetric characteristics across electrodes, ${ }^{35}$ the potential of the anodic peak for dopamine on the $+1.3 \mathrm{~V}$ waveform varied over a range of $40 \mathrm{mV}$. Significant differences were also seen in the peak current ratios between waveforms (Figure 1e). This is likely due to a greater contribution of adsorption to the signal on extended waveforms. As the oxidized form of dopamine (dopamine-o-quinone) adsorbs less strongly than dopamine, it is more likely to desorb before its subsequent reduction, ${ }^{36}$ resulting in an enhanced $i_{\mathrm{p} . \mathrm{a}} / i_{\mathrm{p}, \mathrm{c}}$ ratio.

Phasic Dopamine Concentrations during Continuous ICSS. To address whether the sustained absence of the dopamine signal in early studies was due to insufficient sensitivity, ICSS measurements were repeated with the original $+1.0 \mathrm{~V}$ waveform and compared with measurements with more sensitive voltammetric waveforms $(+1.3 \mathrm{~V}$ and $+1.4 \mathrm{~V})$. Rats were trained to respond on a fixed-ratio 1 (FR1, lever press) schedule for electrical stimulation of the substantia nigra/ ventral tegmental area (SN/VTA) region following previous protocol. ${ }^{19}$ To prevent severe motor responses associated with a

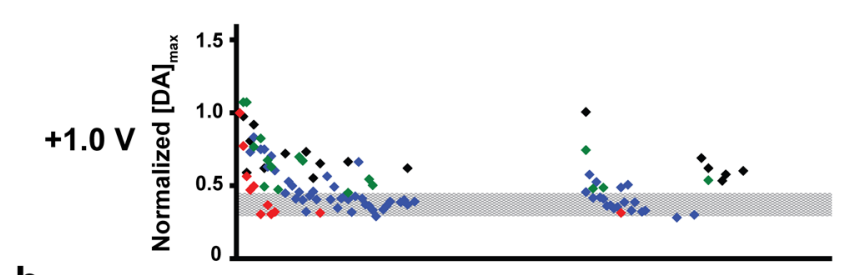

b

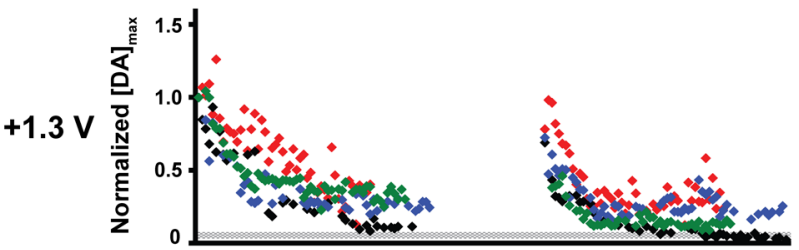

C

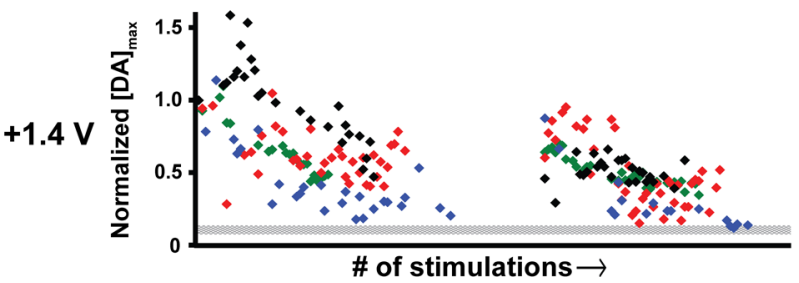

d

ICSS $1 \quad$ (Timeout)

ICSS 2

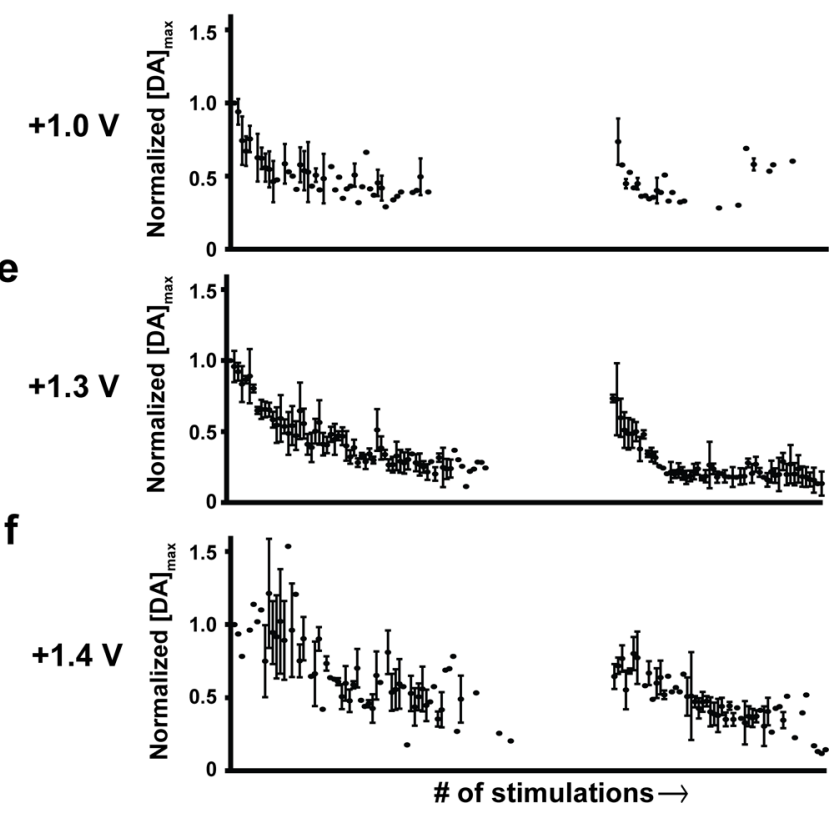

Figure 2. Amplitude of individual dopamine transients during and across ICSS sessions with different waveforms. Dopamine concentrations were normalized to the concentration of the first electrically evoked transient of the first ICSS session for each separate subject. Transients that fell below the within-subject LOD (depicted by gray bar, mean \pm SEM) were discarded. Each waveform had four separate subjects, represented by the four different colors. (a) Measurements on the $+1.0 \mathrm{~V}$ waveform revealed a decrease in dopamine during the first ICSS session and few detectable transients in the subsequent session. (b) Measurements on the $+1.3 \mathrm{~V}$ waveform reveal decreases in dopamine in both ICSS sessions, with the majority of transients detected and quantifiable. (c) Measurements on the $+1.4 \mathrm{~V}$ waveform reveal decreases in dopamine in both ICSS sessions but less consistency between recordings than measurements on the $+1.3 \mathrm{~V}$ waveform. $(\mathrm{d}-\mathrm{f})$ Average concentration profile for $(\mathrm{d})+1.0 \mathrm{~V}$ waveform, $(\mathrm{e})$ $+1.3 \mathrm{~V}$ waveform, and $(\mathrm{f})+1.4 \mathrm{~V}$ waveform. Data points depict mean $\pm \mathrm{SEM}$ at each stimulation. 
the large current intensities used in anesthetized animals (300 $\mu \mathrm{A})$, smaller current intensities were used for ICSS training and recordings $(75-175 \mu \mathrm{A})$. Once trained, each rat was assigned one of the three waveform variations for data acquisition. Stimulation currents were not significantly different between waveforms (one-way ANOVA, $F(2,9)=1.499, p>0.05$ ). A carbon-fiber microelectrode was lowered into the NAc shell in $150 \mu \mathrm{m}$ increments until electrically evoked dopamine release was detected and optimized, ${ }^{33}$ after which experimenterdelivered ("noncontingent") stimulations were administered to establish baseline DA release in the final recording location. Next, rats were allowed to press a lever continuously for a minimum of 50 electrical stimulations. This process was subsequently repeated $30 \mathrm{~min}$ later in a separate behavioral session.

For each ICSS session in each animal, dopamine maximal concentrations following each lever press (evoked by the electrical stimulation, $\left.[\mathrm{DA}]_{\max }\right)$ were determined by PCR and monitored as a function of stimulation number (Supplementary Figure 1). Transients that failed residual analysis at $[\mathrm{DA}]_{\max }$ were considered "invalid", and concentration values were not recorded $(+1.0 \mathrm{~V}$ waveform, $17 \%$ of electrically evoked transients; $+1.3 \mathrm{~V}$ waveform, $2 \%$; $+1.4 \mathrm{~V}$ waveform, $2 \%)$.

While PCA reduces noise, ${ }^{26,27}$ some noise remains in the concentration traces. To ensure that dopamine was being measured at lower concentrations, we evaluated the limit of detection (LOD) for each voltammetric recording session. This was done with the electrode implanted in the brain at the recording locations where ICSS data was collected, but in the absence of electrical stimulation, to ensure that noise levels were similar to those during ICSS. To minimize the effects of short-term electrode drift, data was analyzed in $10 \mathrm{~s}$ intervals for LOD determination. Segments in which spontaneous dopamine transients were apparent in the color plot, or which failed residual analysis, were excluded from these LOD determinations. The apparent "dopamine concentration" for each segment was extracted with PCR, and the average noise of these segments (taken as three times the root-mean-square noise, $n \geq 5$ separate replicates for each subject) was taken as the LOD of the data set. There were significant differences in LODs between waveforms (+1.0 V waveform, $92 \pm 8 \mathrm{nM} ;+1.3$ $\mathrm{V}$ waveform, $31 \pm 4 \mathrm{nM}$; $+1.4 \mathrm{~V}$ waveform, $17 \pm 2 \mathrm{nM}, n=4$; one-way ANOVA, $F(2,9)=17.21, p<0.001)$. Tukey's multiple comparisons post hoc analysis revealed significant differences between +1.0 vs +1.3 and $+1.4(p<0.01$ and 0.001, respectively) but not +1.3 vs $+1.4(p>0.05)$.

Concentrations evoked by electrical stimulation differed between animals, most likely due to differences in placements of the stimulating and working electrodes (concentration of first lever-press induced transient; $355 \pm 66 \mathrm{nM}$, range from 60 to $832 \mathrm{nM})$. These concentrations were not significantly correlated with stimulation current $\left(r^{2}=0.236, p>0.05\right)$. To make reliable comparisons across animals, concentrations and LOD values were normalized for each animal to the concentration of the first electrically evoked ICSS transient in the first behavioral session. Transients that fell below the within-subject LOD were discarded from analysis $(+1.0 \mathrm{~V}$ waveform, $69 \%$ of valid transients; $+1.3 \mathrm{~V}$ waveform, $0.02 \%$; $+1.4 \mathrm{~V}$ waveform, $0 \%$ ). While $[\mathrm{DA}]_{\max }$ decreased with stimulation number with each waveform, the profile of dopamine release differed across waveforms (Figure 2). Measurements with an anodic limit of $+1.0 \mathrm{~V}$ revealed a similar pattern to those seen in the original study (Figure 2a).
Dopamine release was observed early in the first ICSS session; however, the transients quickly decreased to smaller amplitudes, with most stimulations failing to produce dopamine transients exceeding the within-subject LOD (mean \pm SEM represented by gray bar). After a $30 \mathrm{~min}$ timeout, in which the lever and electrical stimulation were unavailable, dopamine release was only observed early in the subsequent ICSS session, while the vast majority of stimulations did not result in observable dopamine release. Due to the paucity of observable dopamine transients, it is difficult to determine a consistent trend for dopamine release during ICSS with this waveform across subjects (Figure $2 \mathrm{~d}$ ).

The enhanced sensitivity of the $+1.3 \mathrm{~V}$ waveform captured dopamine release throughout the entire ICSS recording session, with nearly all electrical stimulations evoking observable events (Figure 2b). Thus, more reliable comparisons of dopamine concentration values both within and across ICSS sessions could be made. The average dopamine concentrations across successive stimulations exhibit a more rapid decay during the second ICSS session (Figure 2e). Regression using a singlephase exponential decay $\left(r^{2}=0.713\right.$ and 0.533 for the two sessions, respectively) revealed a significantly larger rate constant for the second ICSS session, indicating a faster decline $(p<0.0001)$. Correspondingly, the average (normalized) dopamine transient concentration was lower in the second session than in the first (ICSS $1,0.436 \pm 0.025$; ICSS 2, $0.231 \pm 0.017$, two-tailed $t$-test, $t(135)=6.753, p<0.0001)$. Taken together, these data could explain the rapid disappearance of the dopamine signal measured on the $+1.0 \mathrm{~V}$ waveform during the second ICSS session, with the transients more rapidly approaching undetectable values.

Measurements with the $+1.4 \mathrm{~V}$ waveform also consistently resulted in observable dopamine signal release during ICSS (Figure 2c). In all rats $(n=4)$, each electrical stimulation resulted in a detectable dopamine transient, partially due to the lower LODs observed with the $+1.4 \mathrm{~V}$ waveform (average $\sim 17$ $\mathrm{nM})$. However, the concentration profile across stimulations differed compared with measurements on the $+1.3 \mathrm{~V}$ waveform. The average profiles (Figure $2 \mathrm{f})$ fit a single-phase decay $\left(r^{2}=\right.$ 0.408 and 0.478 for the two sessions, respectively) but did not have significantly different rate constants $(p>0.05)$. Nonetheless, the average normalized concentration values were lower in the second ICSS session compared with the first (ICSS 1, 0.684 \pm 0.034 , ICSS 2, $0.457 \pm 0.022$, two-tailed $t$-test, $t(111)=$ 5.174, $p<0.0001)$.

A repeated measures two-way ANOVA was performed to test for differences in press rate between waveform groups and ICSS sessions. No interaction was found between waveform and ICSS session $(F(2,9)=0.690, p>0.05)$. No main effect of waveform $(F(2,9)=1.008, p>0.05)$ or ICSS session $(F(1,9)=$ 1.733, $p>0.05)$ was observed. Therefore, the lower dopamine concentrations during the second ICSS session were not associated with changes in press rates. This is consistent with recent evidence that the amplitude of phasic dopamine release can be dissociated from pressing rate.

Sensitivity Determines Ability to Consistently Monitor Dopamine. To understand why different voltammetric waveforms provide drastically different dopamine release profiles during ICSS, it is necessary to recognize the benefits and limitations of each. Representative traces for the first ICSS session are shown for three separate subjects measured with different waveforms (Figure 3). Representative data indicate the most common features with measurements using the $+1.0 \mathrm{~V}$ 


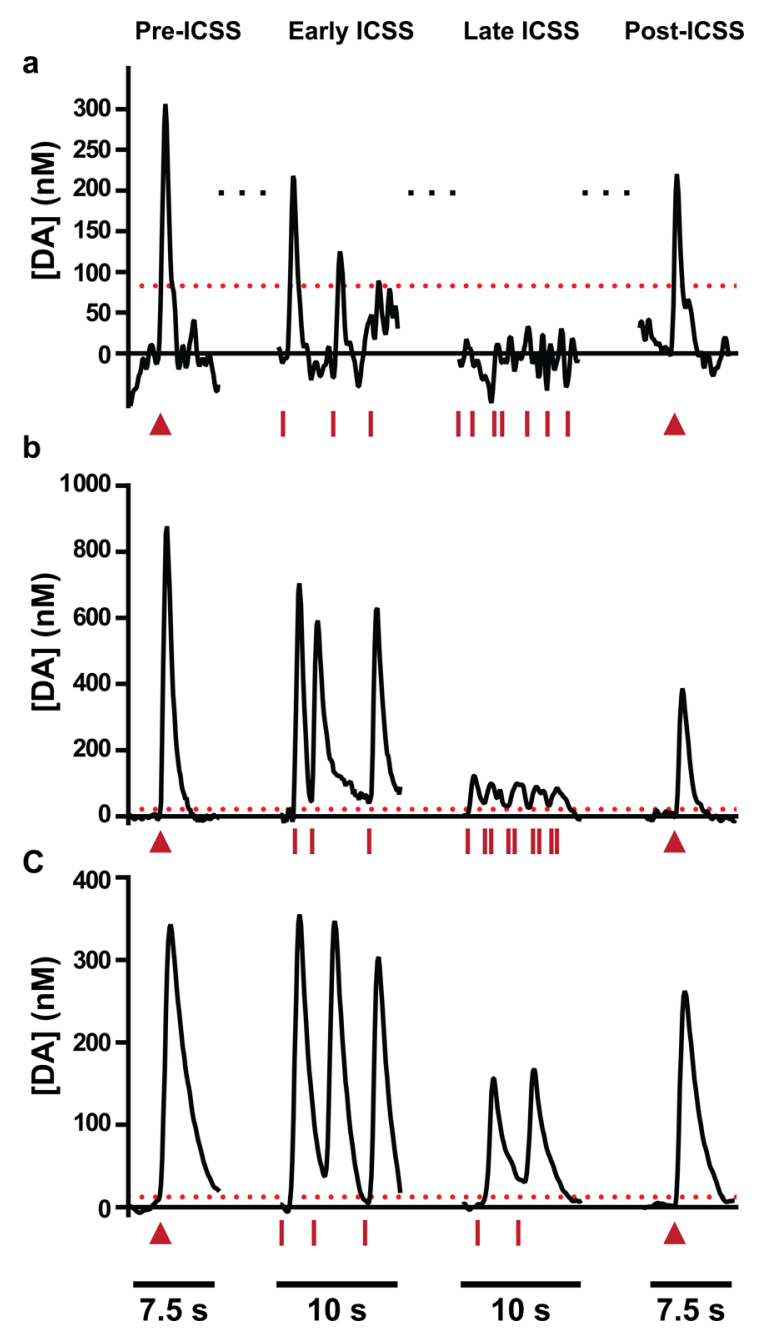

Figure 3. Dopamine concentration versus time traces for representative measurements on each waveform before, during, and after the first ICSS session. Red triangles and red bars represent noncontingent and operant-delivered electrical stimulation, respectively. Red dotted lines indicate LOD for each data set. (a) Measurements on the $+1.0 \mathrm{~V}$ waveform reveal electrically evoked dopamine transients above the LOD $(85 \mathrm{nM})$ before and immediately after ICSS. During early ICSS, dopamine release exceeds the LOD but falls to undetectable levels later in the behavioral session. (b) Measurements on the $+1.3 \mathrm{~V}$ waveform consistently reveal dopamine release above the LOD (21 $\mathrm{nM}$ ) before, during, and immediately after ICSS. Notably, dopamine release falls to much smaller levels late in the ICSS session after prolonged stimulation. (c) Measurements on the $+1.4 \mathrm{~V}$ waveform reveal electrically evoked dopamine transients above the LOD (12 $\mathrm{nM}$ ) before, during, and immediately following ICSS.

waveform (Figure 3a). Noncontingent stimulation ("preICSS") and the first lever press-induced stimulations during ICSS ("early ICSS") evoke sharp dopamine transients that surpass the LOD (red dotted line). However, electrical stimulations near the end of the ICSS session ("late ICSS") do not evoke observable dopamine signals. The inability to detect dopamine release is not due to complete dopamine depletion or electrode failure, because noncontingent stimulation following ICSS ("post-ICSS") resulted in observable dopamine transients (normalized concentration ICSS 1, 0.708 \pm 0.112 ; ICSS $2,0.457 \pm 0.070$ ). Nonetheless, the smaller concentration of dopamine transients after prolonged stim- ulation prevents this waveform from providing a reliable measurement of dopamine release during this task.

Measurements with the $+1.3 \mathrm{~V}$ and $+1.4 \mathrm{~V}$ waveforms, however, were able to consistently monitor dopamine release (Figure 3b,c). While dopamine transients diminish in amplitude during ICSS, the enhanced sensitivity permits the measurement of dopamine transients that would be near or below the LOD on the $+1.0 \mathrm{~V}$ waveform. The wider time course of the transients with these two waveforms compared with the $+1.0 \mathrm{~V}$ waveform is consistent with the observations made in anesthetized animals.

No significant differences were seen between the concentrations evoked by the first-lever press during ICSS and noncontingent stimulation preceding the session (paired twotailed $t$ test, $t(23)=0.7367, p>0.05)$. However, these two signals were significantly correlated across animals and sessions $\left(r^{2}=0.804, p<0.0001\right)$, with larger release to noncontingent stimulation predicting higher concentrations during ICSS.

Effect of Biofouling on Continuous Measurements. The diminished ability to detect dopamine on the $+1.0 \mathrm{~V}$ waveform during the second ICSS session could be related to progressive biofouling of the carbon-fiber microelectrode. The sensitivity of carbon-fiber microelectrodes has been demonstrated previously to decrease upon implantation in the brain. ${ }^{38,39}$ Notably, waveforms with extended anodic limits have been shown be more resistant to biofouling due to active regeneration of the carbon-fiber surface, ${ }^{29}$ which could explain their comparative success in monitoring dopamine during later time points. To investigate this issue, the relative concentration values of the noncontingent stimulations preceding each ICSS session were first compared. On all three waveforms, the electrically evoked signals preceding the second ICSS session were generally lower in amplitude than noncontingent stimulations preceding the first ICSS session $(+1.0 \mathrm{~V}$ waveform, $62.7 \% \pm 14.8 \%$; $+1.3 \mathrm{~V}$ waveform, $68.3 \% \pm 10.3 \%$; $+1.4 \mathrm{~V}$ waveform, $81.9 \% \pm 5 \%)$. While a general trend was observed across anodic limits, no systematic difference was seen between signal recovery and waveform (one-way ANOVA, $F(2,11)=$ $0.815, p>0.05)$. Furthermore, the magnitude of signal depression was not significantly correlated with the time between the two sets of noncontingent stimulations $(43 \pm 2$ $\left.\min ; r^{2}=0.217, p>0.05\right)$ or stimulation current $\left(r^{2}=0.082, p\right.$ $>0.05)$.

Despite the lack of correlation between the timing of noncontingent stimulation and signal depression, the results could be confounded by the variation in implantation time between subjects. Therefore, further biofouling experiments were performed to test the effect of precise implantation times on the sensitivity loss due to in vivo biofouling for each waveform. Carbon-fiber microelectrodes were calibrated before and after 60 or 90 min of implantation in anesthetized rat brain (Table 2). A two-way ANOVA revealed no interaction between

Table 2. Postimplantation Sensitivity for Different Implantation Durations and Waveforms ${ }^{a}$

$\begin{array}{ccc}\text { waveform } & 1 \mathrm{~h}(\%) & 1.5 \mathrm{~h}(\%) \\ +1.0 \mathrm{~V} & 44.5 \pm 6.1 & 37.9 \pm 5.8 \\ +1.3 \mathrm{~V} & 51.4 \pm 4.2 & 51.4 \pm 5.8 \\ +1.4 \mathrm{~V} & 106.4 \pm 12.1 & 108.5 \pm 7.7\end{array}$

${ }^{a}$ Values expressed as mean \pm SEM. $n=5$ for all pairs of waveformduration. 


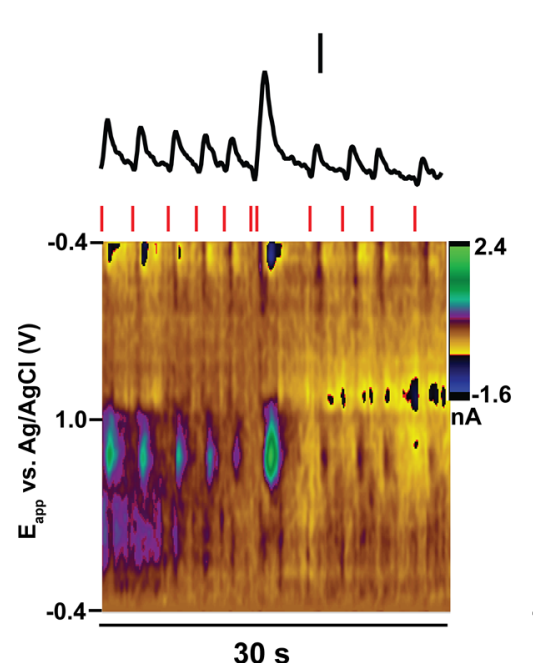

b

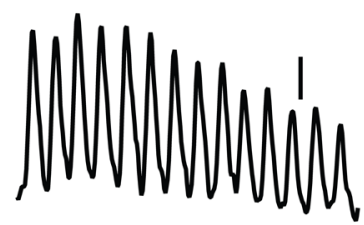

| | | | | | | | | | | | |

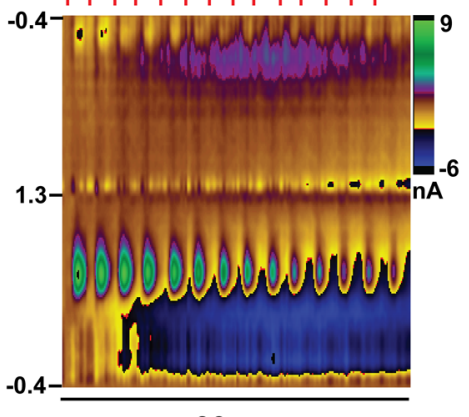

$30 \mathrm{~s}$
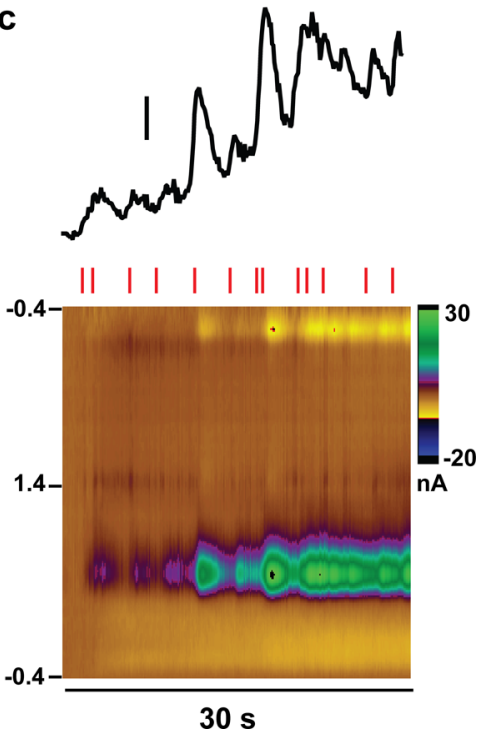

Figure 4. Temporal resolution is diminished with extended anodic limits. (a) Measurements on the $+1.0 \mathrm{~V}$ waveform reveal that electrical stimulation evokes transient increases in dopamine concentration that rapidly return to baseline before subsequent transients. (b) Measurements on the $+1.3 \mathrm{~V}$ waveform typically reveal resolved transients at moderate pressing frequencies, though dopamine concentrations do not always return to baseline before onset of subsequent transients, leading to apparent "facilitations" in the DA signal. (c) Measurements on the $+1.4 \mathrm{~V}$ waveform suffer from drastically diminished temporal resolution. Some cases of moderate pressing rates revealed rising envelopes of DA signal with superimposed transients, making it difficult to ascertain how dopamine dynamics were changing on a stimulation-by-stimulation basis. Scale bars $=250 \mathrm{nM}$.

implantation duration and waveform $(F(2,24)=0.1936 p>$ $0.05)$ or main effect of implant duration $(F(1,24)=0.0621, p>$ $0.05)$ on postcalibration sensitivity. However, there was a main effect of waveform $(F(1,24)=46.37, p<0.0001)$. Tukey's multiple comparisons post hoc revealed significant differences between the $+1.4 \mathrm{~V}$ waveform signal recovery and both the $+1.0 \mathrm{~V}$ and $+1.3 \mathrm{~V}$ waveform at both implant durations $(p>$ $0.0001)$ but not between the $+1.0 \mathrm{~V}$ and $+1.3 \mathrm{~V}$ waveform $(p>$ $0.05)$. Interestingly, the $+1.4 \mathrm{~V}$ waveform maintained preimplantation sensitivity for both implant durations, likely due to continued conditioning of the electrode surface in vivo. Consequently, while the trend in postcalibration sensitivity across waveforms is similar to the trends in restoration of the dopamine signal, the failure to completely restore the signal when measuring with the $+1.4 \mathrm{~V}$ waveform suggests that biofouling is not the sole determinant of the decay in the dopamine signal.

Temporal Resolution Determines Ability to Reliably Quantitate Rapidly Spaced Transients. It has been previously demonstrated that temporal resolution is reduced at extended anodic limits. ${ }^{28}$ Indeed, this is apparent in Figure 3, as electrically evoked dopamine transients measured on extended waveforms, particularly with the $+1.4 \mathrm{~V}$ waveform, are broader than those measured on the $+1.0 \mathrm{~V}$ waveform. This is of particular concern for the continuous ICSS paradigm, which permits the rat to press the lever for electrical stimulation ad libitum, receiving a maximum of one stimulation every 400 ms (i.e., the duration of electrical stimulus). Thus, it is possible in this paradigm for rats to undergo rapid bouts of pressing in which dopamine transients become difficult to resolve due to the finite response time of the carbon-fiber microelectrodes.

To demonstrate how this effect varies across waveforms, three representative 30 second segments of similar pressing rates collected on different waveforms are shown in Figure 4. Lever presses generally induce transients that rapidly return to baseline when measured with the $+1.0 \mathrm{~V}$ waveform (Figure $4 \mathrm{a}$ ).
With a similar press rate, measurements on the $+1.3 \mathrm{~V}$ waveform also result in resolvable transients, but baseline is not always reached between each transient (Figure 4b). When baseline is not reached, an apparent "facilitation" in the signal occurs as previous transients contribute to $[\mathrm{DA}]_{\max }$ for subsequent events (see transients 3-4, Figure 4b). Similar results can be seen for measurements with the $+1.4 \mathrm{~V}$ waveform (data not shown); however, moderate pressing rates occasionally resulted in a rising envelope of dopamine signal with superimposed individual transients (Figure 4c). While digital background subtraction immediately before each transient can partially diminish the contribution of this "envelope" to $[\mathrm{DA}]_{\max }$ it cannot eliminate background rises occurring during the dopamine transients themselves. As a result, these temporally distorted signals can lead to overestimations of $[\mathrm{DA}]_{\max }$ and can make it difficult to ascertain how electrically evoked release changes on a stimulation-by-stimulation basis.

In addition to rising "envelopes" interfering with quantitation of $[\mathrm{DA}]_{\max }$ the rapid nature of continuous ICSS can lead to individual transients becoming unresolvable when stimulations are too narrowly spaced (see presses 6-7 in Figure 4a and presses 9-11 in Figure 4c). The diminished temporal resolution of extended waveforms exacerbates this problem. To investigate this, the percentage of observable transients (i.e., those above the LOD and passing residual analysis) resolved at half-maximum from the preceding transient was determined for each ICSS session on each waveform. If resolved at halfmaximum, it was assumed that the preceding transient will minimally contribute to $[\mathrm{DA}]_{\max }$ of the subsequent transient. Transients that did not meet this criteria were excluded from Figure 2. The ability to resolve transients significantly decreased at extended anodic limits (Figure 5a; one-way ANOVA, $F(2,21)=15.95, p<0.0001)$. Tukey's multiple comparisons post hoc test revealed significant differences in the percent resolution between anodic limits of $+1.0 \mathrm{~V}$ and $+1.4 \mathrm{~V}(p<$ 

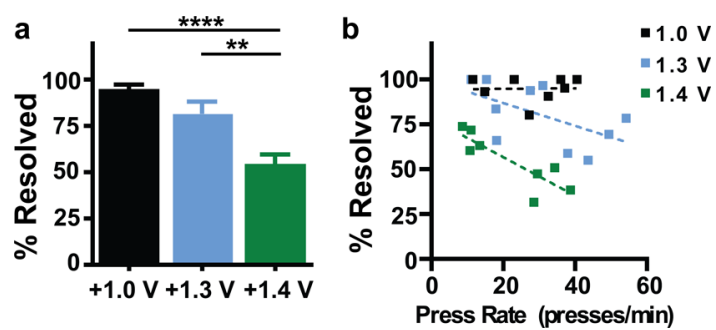

Figure 5. Temporal resolution and its dependence on pressing rate differ across waveforms. (a) The percentage of transients that were resolved at half-maximum from the preceding transient differed among waveforms $(+1.0 \mathrm{~V}$ waveform, $95.0 \% \pm 2.5 \%$; $+1.3 \mathrm{~V}$ waveform, $81.6 \%$ $\pm 6.6 \%$; $+1.4 \mathrm{~V}$ waveform, $54.8 \% \pm 5.4 \%)$. One way ANOVA with Tukey's multiple comparisons, $* * p<0.01$, $* * * * p<0.001$. (b) The effect of press rate on temporal resolution varied across waveforms. The temporal resolution of detectable transients on the $+1.0 \mathrm{~V}$ waveform was relatively insensitive to the range of observed pressing rates. Measurements at higher pressing rates suffered from diminished temporal resolution on the $+1.3 \mathrm{~V}$ waveform, while temporal resolution on the $+1.4 \mathrm{~V}$ waveform was diminished at all pressing rates.

$0.0001)$ and between $+1.3 \mathrm{~V}$ and $+1.4 \mathrm{~V}(p<0.01)$ but not between $+1.0 \mathrm{~V}$ and $+1.3 \mathrm{~V}(p>0.05)$.

However, the resolution of adjacent transients is also impacted by the pressing rate of the animal, since higher pressing rates will result in more narrowly separated dopamine events. Therefore, the percent resolution was compared with the pressing rate for each corresponding ICSS session for each waveform (Figure $5 b$ ). Linear fits to the data show a relatively flat profile for data collected on the $+1.0 \mathrm{~V}$ waveform, indicating that detectable transients are readily resolved at moderate to high pressing rates. The $+1.3 \mathrm{~V}$ waveform performs well at lower pressing rates, but higher press rates result in diminished ability to resolve, and subsequently quantitate, adjacent dopamine transients. This effect is even more pronounced on the $+1.4 \mathrm{~V}$ waveform, where temporal resolution suffers even at slower press rates and fails dramatically at higher pressing rates, where approximately half of transients were unable to be adequately resolved.

Balance of Temporal Resolution and Sensitivity Determines Ability To Monitor Rapid Dopamine Dynamics. Taken together, these data suggest the ability to reliably determine the shape of the dopamine concentration profile during ICSS depends on the trade-off between enhanced sensitivity and diminished temporal resolution. Only $23 \%$ of dopamine transients could be reliably quantified with the +1.0 $\mathrm{V}$ waveform, with a large percentage of presses (56\%) resulting in unobservable events (Figure 2, Table 3). Conversely, measurements on the $+1.3 \mathrm{~V}$ waveform resulted in only one transient that fell below the LOD across all subjects and ICSS sessions. However, a higher percentage of transients (20\%) were unresolvable from preceding transients. This problem was exacerbated on the $+1.4 \mathrm{~V}$ waveform, where nearly half $(45 \%)$ of transients were unable to be resolved. Ultimately, of the three waveforms investigated, the $+1.3 \mathrm{~V}$ waveform was able to provide the best balance of enhanced sensitivity and temporal resolution, quantifying the greatest percentage (78\%) of electrically evoked transients.

\section{DISCUSSION}

This study supports the previous conclusion that continuously elevated phasic dopamine release is not required for maintenance of ICSS behavior. ${ }^{19}$ However, in contrast to the
Table 3. Total Number (and Percentage) of Transients That Were Quantifiable, below the LOD, Unresolved from Preceding Transients, or Failed Residual Analysis across Waveforms $^{a}$

$\begin{array}{ccccc}\text { waveform } & \text { quantifiable } & \text { undetected } & \text { unresolved } & \begin{array}{c}\text { failed } \\ \text { residual }\end{array} \\ +1.0 \mathrm{~V} & 107(23 \%) & 262(56 \%) & 17(4 \%) & 81(17 \%) \\ (n=467) & & & & \\ +1.3 \mathrm{~V} & 381(78 \%) & 1(0.02 \%) & 96(20 \%) & 10(2 \%) \\ (n=488) & & & & 7(2 \%) \\ +1.4 \mathrm{~V} & 236(54 \%) & 0(0 \%) & 198(45 \%) & 7(n=441) \\ & & & & \end{array}$

$a_{n}=$ total number of transients for all ICSS sessions measured with each waveform.

original findings, the use of more sensitive waveforms revealed that dopamine transients do not completely disappear during this task but rather diminish to smaller values undetectable in previous investigations. Notably, continuous ICSS is a unique behavioral paradigm in which the ability to resolve narrowly spaced events is crucial. Thus, the enhanced ability of the +1.3 $\mathrm{V}$ waveform to detect electrically evoked transients throughout ICSS while maintaining moderate temporal resolution supports its use as the standard waveform for measurements in awake animals with narrowly spaced dopamine events. However, the enhanced sensitivity of $+1.4 \mathrm{~V}$ waveform may be useful for studying behavioral paradigms in which temporal resolution is unnecessary for understanding the voltammetric data (e.g., studies without narrowly spaced cues or behavioral responses). Nonetheless, its use to study rapid behaviors like ICSS could lead to erroneous conclusions about dopamine dynamics; indeed, the $+1.4 \mathrm{~V}$ waveform revealed "rises" in dopamine concentrations during ICSS (Figure 4c) similar to those seen in microdialysis experiments, rather than a decay in dopamine release as suggested by the other waveforms. This reduced temporal resolution could alter concentration prediction for moderately resolved release events (i.e., resolved at halfmaximum), with tailing currents from preceding transients augmenting concentration values for subsequent events.

Potential offsets are often used in voltammetric recordings in awake animals because of biofouling of chronically implanted reference electrodes that results in an altered potential. The offsets are often quite large, typically $\sim 200 \mathrm{mV}^{25}$ The use of offsets can lead to an unwanted extended anodic limit, resulting in altered electrode performance. Accordingly, acute reference electrodes were used in this study to reliably compare waveforms. As shown here and elsewhere, the voltammetric waveform, including any offsets, is an important experimental consideration that can affect sensitivity, temporal resolution, and electrode stability. ${ }^{32}$

A difference between this study and that of Garris et al. is the stimulus pulse duration ( 2 and $1 \mathrm{~ms}$ pulses, respectively). Longer pulse widths promote slightly greater dopamine release when stimulation current remains constant. ${ }^{40}$ However, longer pulse widths have more pronounced motor effects during electrical stimulation, which can limit rapid response rates during ICSS. The moderate press rates in this study with longer pulse widths made it possible to resolve individual transients and study changes in phasic dopamine release over time. Shorter pulse widths could permit more rapid pressing; however, this may result in unresolved transients, particularly with extended waveforms. It has been hypothesized that extracellular dopaminergic tone is a function of summated 
phasic dopamine transients. ${ }^{41}$ Thus, it is possible that these signals (i.e., unresolved dopamine transients) would begin to resemble microdialysis signals for rapid pressing, ${ }^{17}$ in which a gradual rise in dopamine is succeeded by a fall in dopamine levels during continuous pressing as the summated transients begin to decay in amplitude (see Figure 4c).

In previous work, a three component model (short-term facilitation, short-term depression, and long-term depression) was developed that predicts dopamine concentrations during isolated and rapid stimulation patterns. ${ }^{42,43}$ The time constant for recovery from long-term depression was predicted to be 12-15 min, and studies in anesthetized rats show full recovery on the order of $30 \mathrm{~min}^{44}$ However, the $30 \mathrm{~min}$ timeout in this study was insufficient for dopamine to return to pre-ICSS levels. Indeed, dopamine release often remained attenuated, which could not be solely attributed to biofouling of the electrode, suggesting that this model has not captured all longterm factors controlling dopamine release. This could be due to depletion of releasable vesicles or a decreased rate of dopamine synthesis. Alternatively, dopamine VTA neurons are thought to be activated transsynaptically during electrical stimulation via ionotropic glutamate receptors; ${ }^{13}$ this mechanism could be altered. For example, it has been shown that the subunit composition of AMPA receptors in the VTA can change after repetitive brain stimulation. ${ }^{45}$ Recent studies have shown that optogenetic stimulation of glutamatergic neurons that form synapses on dopamine neurons within the VTA is sufficient to promote ICSS $^{46}$ and evoke dopamine release in the NAc. ${ }^{47}$

ICSS can be learned very quickly. In fact, we have previously shown that dopamine release and ICSS behavior are acquired and reach stable response within 200 lever presses. ${ }^{21}$ Therefore, it is unlikely that differences in training history between subjects were a significant source of variability in the decay of dopamine release between and across sessions. However, future longitudinal studies of dopamine release during ICSS could investigate whether these dopamine profiles change over extended training.

While this study did not manipulate phasic dopamine release to test its effect on behavior, it was found that even as the concentration of dopamine transients in the NAc fell across ICSS sessions, subjects continued to respond for ICSS at similar rates. Therefore, the original assertion that consistently elevated phasic dopamine release is unnecessary for the maintenance of ICSS remains valid. Nevertheless, a full dissociation of dopamine release from ICSS behavior seems unlikely. A few possibilities for the role of electrically evoked dopamine release in ICSS remain. First, the high dopamine concentrations evoked by early stimulation may be required for acquisition of this task via corticostriatal synaptic plasticity that promotes learning ${ }^{48}$ but may be unnecessary for maintenance. However, pharmacological manipulation of dopamine in welltrained animals can still alter behavior, ${ }^{2,7,8}$ which contradicts this view. Second, the low steady-state dopamine concentrations seen after repeated response in this study, as well as the slower increases in dopaminergic tone demonstrated with microdialysis, ${ }^{16-18}$ may be sufficient to activate high-affinity D2 receptors on striatal medium spiny neurons, which inhibits their activity. ${ }^{49}$ Activation of D2-expressing medium spiny neurons is aversive. $^{50}$ Therefore, it is possible that the relatively low dopamine concentrations after continuous pressing remain vital for inhibiting these cells to prevent activation of circuitry that could compete with the primary reward pathway activated by ICSS. The mechanism we prefer is that dopamine mediates responses to cues predicting reward availability. Although the visual cue and lever were both continually present here, making it impossible to separate their contributions, elsewhere using a delayed lever availability paradigm we have shown that dopamine responses to cues during ICSS modulate D2containing medium spiny neurons. ${ }^{51}$ Indeed, we found that D1-mediated responses occurred near the time of the stimulation, whereas responses after the cue were mediated by $\mathrm{D} 2$ receptors. Therefore, there is evidence that dopamine is important for both cue and operant responses. Ultimately, future studies involving pharmacological manipulations of dopamine during ICSS with FSCV measurements will further elucidate the relationship between phasic dopamine release and ICSS behavior.

\section{METHODS}

Animals. Male Sprague-Dawley rats $(250-450 \mathrm{~g})$ from Charles River (Wilmington, MA, USA) were housed individually on a $12 / 12 \mathrm{~h}$ light/dark cycle. Rats were given access to water and food chow ad libitum. Animal procedures were approved by the UNC-Chapel Hill Institutional Animal Care and Use Committee (IACUC).

Surgery. Animals were anesthetized using isoflurane (1.5-4\%). Guide cannulas for the working electrode (Bioanalytical Systems, West Lafayette, IN) were implanted above the NAc shell using stereotaxic coordinates (AP $+1.7 \mathrm{~mm}, \mathrm{ML}+0.8 \mathrm{~mm}$, DV $2.5 \mathrm{~mm}$ ). A separate guide cannula was implanted in the contralateral hemisphere to allow experiment-day lowering of a fresh $\mathrm{Ag} / \mathrm{AgCl}$ reference electrode. A bipolar stimulating electrode (Plastics One, Roanoke, VA) was positioned above the ipsilateral VTA (AP $-5.2 \mathrm{~mm}, \mathrm{ML}+1.0 \mathrm{~mm}$, DV $-8.4-8.8 \mathrm{~mm}$ ventral from skull surface). Stainless steel screws and dental cement were used to secure the cannulas and stimulating electrode to the skull surface. Animals were given a minimum of 3 days of postsurgery recovery before behavioral training.

For biofouling experiments, surgical preparation differed slightly. Animals were anesthetized with urethane, and no stimulating electrode or guide cannulas were used. Fresh carbon-fiber and $\mathrm{Ag} / \mathrm{AgCl}$ electrodes were used for implantation.

Behavior. Rats were trained in intracranial self-stimulation following protocol described previously. ${ }^{19}$ Rats were placed in plexiglass operant chambers (Med Associates Inc., St. Albans, VT. USA) and connected to head-mounted voltammetric amplifier attached to a commutator (Crist Instrument Co., Hagerstown, MD, USA) that permitted movement within the behavioral chamber. Stimulation current was applied through an optically isolated current source (NeuroLog NL-800, Medical Systems, Greenvale, NY, USA) and adjusted to the maximal current that did not evoke strong motor responses that would prevent reliable behavior (24 biphasic pulses, 60 $\mathrm{Hz}, 2 \mathrm{~ms}$ pulses, $75-175 \mu \mathrm{A}$ ). Following current adjustment, the behavioral session was initiated with the onset of white noise, cue light illumination, and lever extension into the chamber. All behavioral events were controlled with a MedAssociates system. Rats were primed with electrical stimulation as they approached the lever until they learned to respond (lever press; fixed-ratio 1, FR1) for selfadministered electrical stimulation. Care was taken to minimize the number of noncontingent stimulations, and noncontingent stimulation was not administered during training once rats had acquired ICSS (i.e., spent entirety of training session responding for ICSS). Rats were trained for a minimum of two sessions per day for a minimum of 3 days (maximum of five). Occasionally, rats were trained for a third session during the first 2 days of training. The lever was retracted for 30 min between behavioral sessions. All rats used for voltammetric recordings acquired and maintained ICSS (i.e., pressed for the entire duration of lever presentation) for the final two testing days without need for noncontingent stimulations.

FSCV. Glass-sealed carbon-fiber microelectrodes $(90-110 \mu \mathrm{m}$ in length) were lowered into the NAc through micromanipulators placed in the implanted guide cannula. On experiment day, freshly coated $\mathrm{Ag} /$ $\mathrm{AgCl}$ reference electrodes were implanted into the contralateral 
hemisphere. Electrodes were cycled (approximately $15 \mathrm{~min}$ at $60 \mathrm{~Hz}$, $15 \mathrm{~min}$ at $10 \mathrm{~Hz}$ ) before electrochemical measurements to minimize the contribution of electrode drift to the signal. All waveforms employed the same scan rate $(400 \mathrm{~V} / \mathrm{s})$ and holding potential $(-0.4$ $\mathrm{V})$, while anodic limits varied $(+1.0,+1.3$, and $+1.4 \mathrm{~V})$. Dopamine release was monitored for a minimum of 50 electrical stimulations per ICSS session in all animals. The session duration required to meet this criteria varied, depending on press rate (mean $167 \mathrm{~s}$, range from 89 to $318 \mathrm{~s})$.

For FSCV recordings in anesthetized animals $(n=8)$, stimulated release events ( $300 \mu \mathrm{A}, 24$ pulses) were measured in the NAc. For within-subject comparisons, stimulated release was measured with all three waveforms $(+1.0,+1.3$, and $+1.4 \mathrm{~V} ; n=6$ stimulations per waveform) in a sequential manner. The electrode was cycled (15 min at $60 \mathrm{~Hz}, 15 \mathrm{~min}$ at $10 \mathrm{~Hz}$ ) each time a new waveform was employed.

Data was collected using HDCV programming (UNC-Chapel Hill, NC, USA) ${ }^{52}$ built in LabView (National Instruments, Austin, TX, USA). Voltammetric current was transduced through locally constructed UEI potentiostat instrumentation (UNC Electronics Facility). Data was digitally filtered (4th order low pass Bessel, 2 $\mathrm{kHz}$ cutoff).

Data Analysis. Dopamine concentrations were predicted using principal component regression (PCR) using residual analysis following previously established protocol and software. ${ }^{24,27,52}$ Training sets were built using dopamine and $\mathrm{pH}$ standards recorded in vivo postexperiment from the same electrode and recording location as the collected data. ${ }^{35}$ Dopamine transients for which $Q_{t}$ exceeded $Q_{\alpha}$ at $[D A]_{\max }$ were excluded from data analysis. Individual release events were analyzed by aligning the voltammetric background to the time of stimulation (preceding the stimulation by $0-2 \mathrm{~s}$ ) for each individual event, minimizing the interference from electrode drift or additive $\mathrm{pH}$ changes from successive stimulation that would exceed the training set range. In a few longer behavioral trials, the number of transients analyzed was limited to the first 70 electrical stimulations to keep data set sizes comparable.

To ensure that measurement of low concentration dopamine transients was reliable, a limit of detection (LOD) was calculated for each separate electrode. Data were collected at the same recording site as ICSS measurements, but without electrical stimulation, to estimate noise levels. The LOD was established as 3 times RMS in the chemometrized dopamine signal.

Statistical Analysis. Results are presented as mean \pm standard error. Statistical tests were performed in GraphPad Software, with results considered significant if $p<0.05$. One-way ANOVA was used for comparisons between the three different waveforms. Repeated measures one-way ANOVA was used when the outcomes were quantitative, independent variables were nominal, and multiple observations were made with each unit. Two-way repeated measures ANOVA was conducted to test the effect of two independent variables on a single dependent variable. If significant differences were found for any ANOVA test, Tukey's multiple comparisons post hoc test was used to make pairwise comparisons. Two-tailed $t$ tests were used to compare the average concentrations between the two ICSS sessions.

Flow Cell Analysis. External calibration factors were determined using flow injection analysis. All dopamine solutions were prepared in TRIS buffer $\left(3.25 \mathrm{mM} \mathrm{KCl}, 1.2 \mathrm{mM} \mathrm{CaCl} 2 \cdot 2 \mathrm{H}_{2} \mathrm{O}, 1.2 \mathrm{mM} \mathrm{MgCl}\right.$. $6 \mathrm{H}_{2} \mathrm{O}, 2.0 \mathrm{mM} \mathrm{Na}_{2} \mathrm{SO}_{4}, 1.25 \mathrm{mM} \mathrm{NaH}_{2} \mathrm{PO}_{4} \cdot \mathrm{H}_{2} \mathrm{O}, 140 \mathrm{mM} \mathrm{NaCl}, 15$ $\mathrm{mM}$ Trizma $\mathrm{HCl}$ ) adjusted to $\mathrm{pH} 7.4$ with $\mathrm{NaOH}$. Dopamine solutions were bubbled under $\mathrm{N}_{2}$ to prevent oxidative degradation of dopamine during successive calibrations. For sensitivity factor measurements between waveforms, a physiological range of dopamine concentrations (50 nM to $2 \mu \mathrm{M}$ ) was used. For biofouling measurements, point calibrations at $1 \mu \mathrm{M}$ for both pre- and postcalibration were used. All calibration currents were normalized by electrode length to $100 \mu \mathrm{m}$.

\section{ASSOCIATED CONTENT}

Supporting Information

The Supporting Information is available free of charge on the ACS Publications website at DOI: 10.1021/acschemneuro.6b00142.

Figure detailing how ICSS data was analyzed with background subtraction and residual analysis (PDF)

\section{AUTHOR INFORMATION}

\section{Author Contributions}

N.T.R. and R.M.W. conceived the study. N.T.R. and E.S.B. performed in vivo experiments. J.A.J. performed calibration. N.T.R. analyzed data. R.M.W. and N.T.R. wrote the manuscript. All authors approved the final version of the manuscript.

\section{Funding}

Authors were supported by the National Institute of Health (Grant DA010900).

\section{Notes}

The authors declare no competing financial interest.

\section{REFERENCES}

(1) Olds, J., and Milner, P. (1954) Positive reinforcement produced by electrical stimulation of septal area and other regions of rat brain. J. Comp. Physiol. Psychol. 47, 419-427.

(2) Carlezon, W. A., Jr., and Chartoff, E. H. (2007) Intracranial selfstimulation (ICSS) in rodents to study the neurobiology of motivation. Nat. Protoc. 2, 2987-2995.

(3) Fulton, S., Woodside, B., and Shizgal, P. (2000) Modulation of brain reward circuitry by leptin. Science 287, 125-128.

(4) Phillips, A. G., and Fibiger, H. C. (1978) The role of dopamine in maintaining intracranial self-stimulation in the ventral tegmentum, nucleus accumbens, and medial prefrontal cortex. Can. J. Psychol 32, 58-66.

(5) Fibiger, H. C., LePiane, F. G., Jakubovic, A., and Phillips, A. G. (1987) The role of dopamine in intracranial self-stimulation of the ventral tegmental area. J. Neurosci. 7, 3888-3896.

(6) Corbett, D., and Wise, R. A. (1980) Intracranial self-stimulation in relation to the ascending dopaminergic systems of the midbrain: a moveable electrode mapping study. Brain Res. 185, 1-15.

(7) Wise, R. A. (1996) Addictive drugs and brain stimulation reward. Annu. Rev. Neurosci. 19, 319-340.

(8) Steinberg, E. E., Boivin, J. R., Saunders, B. T., Witten, I. B., Deisseroth, K., and Janak, P. H. (2014) Positive reinforcement mediated by midbrain dopamine neurons requires D1 and D2 receptor activation in the nucleus accumbens. PLoS One 9, e94771.

(9) Gallistel, C. R., Shizgal, P., and Yeomans, J. S. (1981) A portrait of the substrate for self-stimulation. Psychol Rev. 88, 228-273.

(10) Yeomans, J. S., Maidment, N. T., and Bunney, B. S. (1988) Excitability properties of medial forebrain bundle axons of A9 and A10 dopamine cells. Brain Res. 450, 86-93.

(11) Bielajew, C., and Shizgal, P. (1986) Evidence implicating descending fibers in self-stimulation of the medial forebrain bundle. $J$. Neurosci. 6, 919-929.

(12) Yeomans, J., and Baptista, M. (1997) Both nicotinic and muscarinic receptors in ventral tegmental area contribute to brainstimulation reward. Pharmacol., Biochem. Behav. 57, 915-921.

(13) Sombers, L. A., Beyene, M., Carelli, R. M., and Wightman, R. M. (2009) Synaptic overflow of dopamine in the nucleus accumbens arises from neuronal activity in the ventral tegmental area. J. Neurosci. $29,1735-1742$.

(14) Witten, I. B., Steinberg, E. E., Lee, S. Y., Davidson, T. J., Zalocusky, K. A., Brodsky, M., Yizhar, O., Cho, S. L., Gong, S., Ramakrishnan, C., Stuber, G. D., Tye, K. M., Janak, P. H., and Deisseroth, K. (2011) Recombinase-driver rat lines: tools, techniques, 
and optogenetic application to dopamine-mediated reinforcement. Neuron 72, 721-733.

(15) Berrios, J., Stamatakis, A. M., Kantak, P. A., McElligott, Z. A., Judson, M. C., Aita, M., Rougie, M., Stuber, G. D., and Philpot, B. D. (2016) Loss of UBE3A from TH-expressing neurons suppresses GABA co-release and enhances VTA-NAc optical self-stimulation. Nat. Commun. 7, 10702.

(16) You, Z. B., Chen, Y. Q., and Wise, R. A. (2001) Dopamine and glutamate release in the nucleus accumbens and ventral tegmental area of rat following lateral hypothalamic self-stimulation. Neuroscience 107, 629-639.

(17) Hernandez, G., Hamdani, S., Rajabi, H., Conover, K., Stewart, J., Arvanitogiannis, A., and Shizgal, P. (2006) Prolonged rewarding stimulation of the rat medial forebrain bundle: neurochemical and behavioral consequences. Behav. Neurosci. 120, 888-904.

(18) Fiorino, D. F., Coury, A., Fibiger, H. C., and Phillips, A. G. (1993) Electrical stimulation of reward sites in the ventral tegmental area increases dopamine transmission in the nucleus accumbens of the rat. Behav. Brain Res. 55, 131-141.

(19) Garris, P. A., Kilpatrick, M., Bunin, M. A., Michael, D., Walker, Q. D., and Wightman, R. M. (1999) Dissociation of dopamine release in the nucleus accumbens from intracranial self-stimulation. Nature $398,67-69$.

(20) Kilpatrick, M. R., Rooney, M. B., Michael, D. J., and Wightman, R. M. (2000) Extracellular dopamine dynamics in rat caudate-putamen during experimenter-delivered and intracranial self-stimulation. Neuroscience 96, 697-706.

(21) Owesson-White, C. A., Cheer, J. F., Beyene, M., Carelli, R. M., and Wightman, R. M. (2008) Dynamic changes in accumbens dopamine correlate with learning during intracranial self-stimulation. Proc. Natl. Acad. Sci. U. S. A. 105, 11957-11962.

(22) Kishida, K. T., Saez, I., Lohrenz, T., Witcher, M. R., Laxton, A. W., Tatter, S. B., White, J. P., Ellis, T. L., Phillips, P. E., and Montague, P. R. (2016) Subsecond dopamine fluctuations in human striatum encode superposed error signals about actual and counterfactual reward. Proc. Natl. Acad. Sci. U. S. A. 113, 200-205.

(23) Yorgason, J. T., Espana, R. A., and Jones, S. R. (2011) Demon voltammetry and analysis software: analysis of cocaine-induced alterations in dopamine signaling using multiple kinetic measures. $J$. Neurosci. Methods 202, 158-164.

(24) Heien, M. L., Johnson, M. A., and Wightman, R. M. (2004) Resolving neurotransmitters detected by fast-scan cyclic voltammetry. Anal. Chem. 76, 5697-5704.

(25) Heien, M. L., Khan, A. S., Ariansen, J. L., Cheer, J. F., Phillips, P. E., Wassum, K. M., and Wightman, R. M. (2005) Real-time measurement of dopamine fluctuations after cocaine in the brain of behaving rats. Proc. Natl. Acad. Sci. U. S. A. 102, 10023-10028.

(26) Keithley, R. B., Carelli, R. M., and Wightman, R. M. (2010) Rank estimation and the multivariate analysis of in vivo fast-scan cyclic voltammetric data. Anal. Chem. 82, 5541-5551.

(27) Keithley, R. B., Heien, M. L., and Wightman, R. M. (2009) Multivariate concentration determination using principal component regression with residual analysis. TrAC, Trends Anal. Chem. 28, 11271136.

(28) Heien, M. L., Phillips, P. E., Stuber, G. D., Seipel, A. T., and Wightman, R. M. (2003) Overoxidation of carbon-fiber microelectrodes enhances dopamine adsorption and increases sensitivity. Analyst 128, 1413-1419.

(29) Takmakov, P., Zachek, M. K., Keithley, R. B., Walsh, P. L., Donley, C., McCarty, G. S., and Wightman, R. M. (2010) Carbon microelectrodes with a renewable surface. Anal. Chem. 82, 2020-2028.

(30) Rebec, G. V., Christensen, J. R., Guerra, C., and Bardo, M. T. (1997) Regional and temporal differences in real-time dopamine efflux in the nucleus accumbens during free-choice novelty. Brain Res. 776, 61-67.

(31) Garris, P. A., Christensen, J. R., Rebec, G. V., and Wightman, R. M. (1997) Real-time measurement of electrically evoked extracellular dopamine in the striatum of freely moving rats. J. Neurochem. 68, 152161.
(32) Keithley, R. B., Takmakov, P., Bucher, E. S., Belle, A. M., Owesson-White, C. A., Park, J., and Wightman, R. M. (2011) Higher sensitivity dopamine measurements with faster-scan cyclic voltammetry. Anal. Chem. 83, 3563-3571.

(33) Wightman, R. M., Heien, M. L., Wassum, K. M., Sombers, L. A., Aragona, B. J., Khan, A. S., Ariansen, J. L., Cheer, J. F., Phillips, P. E., and Carelli, R. M. (2007) Dopamine release is heterogeneous within microenvironments of the rat nucleus accumbens. Eur. J. Neurosci 26, 2046-2054.

(34) Bard, A. J., and Faulkner, L. R. (2001) Electrochemical methods: fundamentals and applications, 2nd ed., Wiley, New York.

(35) Rodeberg, N. T., Johnson, J. A., Cameron, C. M., Saddoris, M. P., Carelli, R. M., and Wightman, R. M. (2015) Construction of Training Sets for Valid Calibration of in Vivo Cyclic Voltammetric Data by Principal Component Analysis. Anal. Chem. 87, 11484.

(36) Bath, B. D., Michael, D. J., Trafton, B. J., Joseph, J. D., Runnels, P. L., and Wightman, R. M. (2000) Subsecond adsorption and desorption of dopamine at carbon-fiber microelectrodes. Anal. Chem. $72,5994-6002$.

(37) Cossette, M. P., Conover, K., and Shizgal, P. (2016) The neural substrates for the rewarding and dopamine-releasing effects of medial forebrain bundle stimulation have partially discrepant frequency responses. Behav. Brain Res. 297, 345-358.

(38) Logman, M. J., Budygin, E. A., Gainetdinov, R. R., and Wightman, R. M. (2000) Quantitation of in vivo measurements with carbon fiber microelectrodes. J. Neurosci. Methods 95, 95-102.

(39) Singh, Y. S., Sawarynski, L. E., Dabiri, P. D., Choi, W. R, and Andrews, A. M. (2011) Head-to-head comparisons of carbon fiber microelectrode coatings for sensitive and selective neurotransmitter detection by voltammetry. Anal. Chem. 83, 6658-6666.

(40) Park, J., Takmakov, P., and Wightman, R. M. (2011) In vivo comparison of norepinephrine and dopamine release in rat brain by simultaneous measurements with fast-scan cyclic voltammetry. $J$. Neurochem. 119, 932-944.

(41) Owesson-White, C. A., Roitman, M. F., Sombers, L. A., Belle, A. M., Keithley, R. B., Peele, J. L., Carelli, R. M., and Wightman, R. M. (2012) Sources contributing to the average extracellular concentration of dopamine in the nucleus accumbens. J. Neurochem. 121, 252-262.

(42) Montague, P. R., McClure, S. M., Baldwin, P. R., Phillips, P. E., Budygin, E. A., Stuber, G. D., Kilpatrick, M. R., and Wightman, R. M. (2004) Dynamic gain control of dopamine delivery in freely moving animals. J. Neurosci. 24, 1754-1759.

(43) Kita, J. M., Parker, L. E., Phillips, P. E., Garris, P. A., and Wightman, R. M. (2007) Paradoxical modulation of short-term facilitation of dopamine release by dopamine autoreceptors. $J$. Neurochem. 102, 1115-1124.

(44) Michael, A. C., Ikeda, M., and Justice, J. B., Jr. (1987) Mechanisms contributing to the recovery of striatal releasable dopamine following MFB stimulation. Brain Res. 421, 325-335.

(45) Carlezon, W. A., Jr., Todtenkopf, M. S., McPhie, D. L., Pimentel, P., Pliakas, A. M., Stellar, J. R., and Trzcinska, M. (2001) Repeated exposure to rewarding brain stimulation downregulates GluR1 expression in the ventral tegmental area. Neuropsychopharmacology $25,234-241$.

(46) Wang, H. L., Qi, J., Zhang, S., Wang, H., and Morales, M. (2015) Rewarding Effects of Optical Stimulation of Ventral Tegmental Area Glutamatergic Neurons. J. Neurosci. 35, 15948-15954.

(47) Qi, J., Zhang, S., Wang, H. L., Wang, H., de Jesus Aceves Buendia, J., Hoffman, A. F., Lupica, C. R., Seal, R. P., and Morales, M. (2014) A glutamatergic reward input from the dorsal raphe to ventral tegmental area dopamine neurons. Nat. Commun. 5, 5390.

(48) Reynolds, J. N., Hyland, B. I., and Wickens, J. R. (2001) A cellular mechanism of reward-related learning. Nature 413, 67-70.

(49) Calipari, E. S., Bagot, R. C., Purushothaman, I., Davidson, T. J., Yorgason, J. T., Pena, C. J., Walker, D. M., Pirpinias, S. T., Guise, K. G., Ramakrishnan, C., Deisseroth, K., and Nestler, E. J. (2016) In vivo imaging identifies temporal signature of D1 and D2 medium spiny neurons in cocaine reward. Proc. Natl. Acad. Sci. U. S. A. 113, 27262731. 
(50) Kravitz, A. V., Tye, L. D., and Kreitzer, A. C. (2012) Distinct roles for direct and indirect pathway striatal neurons in reinforcement. Nat. Neurosci. 15, 816-818.

(51) Owesson-White, C., Belle, A. M., Herr, N. R., Peele, J., Gowrishankar, P., Carelli, R. M., and Wightman, R. M. (2016) Cueevoked dopamine release rapidly modulates $\mathrm{D} 2$ neurons in the nucleus accumbens during motivated behavior. J. Neurosci. 36, 6011.

(52) Bucher, E. S., Brooks, K., Verber, M. D., Keithley, R. B., Owesson-White, C., Carroll, S., Takmakov, P., McKinney, C. J., and Wightman, R. M. (2013) Flexible software platform for fast-scan cyclic voltammetry data acquisition and analysis. Anal. Chem. 85, 1034410353. 